\title{
Energy decay of dissipative plate equations with memory-type boundary conditions
}

\author{
Muhammad I. Mustafa \\ Department of Mathematics, University of Sharjah, Sharjah 27272, United Arab Emirates \\ E-mail: mmustafa@sharjah.ac.ae
}

\begin{abstract}
In this paper we consider a plate equation with internal feedback and viscoelastic damping localized on a part of the boundary. Without imposing restrictive assumptions on the time-dependent frictional damping, we establish an explicit and general decay rate result that allows a wider class of relaxation functions and generalizes previous results existing in the literature.
\end{abstract}

Keywords: plate equation, general decay, viscoelastic damping, relaxation function, frictional damping

\section{Introduction}

In this paper we are concerned with the following problem

$$
\begin{aligned}
& u_{t t}+\Delta^{2} u+\theta(t) h\left(u_{t}\right)=0 \quad \text { in } \Omega \times(0, \infty), \\
& u=\frac{\partial u}{\partial v}=0 \quad \text { on } \Gamma_{0} \times(0, \infty), \\
& -u+\int_{0}^{t} g_{1}(t-s) \beta_{2} u(s) d s=0 \quad \text { on } \Gamma_{1} \times(0, \infty), \\
& \frac{\partial u}{\partial v}+\int_{0}^{t} g_{2}(t-s) \beta_{1} u(s) d s=0 \quad \text { on } \Gamma_{1} \times(0, \infty), \\
& u(x, y, 0)=u_{0}(x, y), \quad u_{t}(x, y, 0)=u_{1}(x, y) \quad \text { in } \Omega,
\end{aligned}
$$

which is a Kirchhoff plates equation with internal frictional damping and memory conditions at a part of the boundary. Here $\Omega$ is a bounded domain of $\mathbb{R}^{2}$ with a smooth boundary $\partial \Omega=\Gamma_{0} \cup \Gamma_{1}, v=\left(v_{1}, v_{2}\right)$ is the unit outward normal to $\partial \Omega, \eta=\left(-v_{2}, v_{1}\right)$ is the unit tangent positively oriented on $\partial \Omega$, the integral terms in (1.3) and (1.4) are the memories responsible for the viscoelastic damping where $g_{1}, g_{2}$ are positive functions called the relaxation functions, $\theta$ is a time dependent coefficient of the frictional damping, and $h$ is a specific function. We are denoting by $\beta_{1}, \beta_{2}$ the following differential operators:

$$
\beta_{1} u=\Delta u+(1-\mu) B_{1} u, \quad \beta_{2} u=\frac{\partial \Delta u}{\partial v}+(1-\mu) \frac{\partial B_{2} u}{\partial \eta}
$$


where

$$
B_{1} u=2 v_{1} v_{2} u_{x y}-v_{1}^{2} u_{y y}-v_{2}^{2} u_{x x}, \quad B_{2} u=\left(v_{1}^{2}-v_{2}^{2}\right) u_{x y}+v_{1} v_{2}\left(u_{y y}-u_{x x}\right)
$$

and $\mu \in\left(0, \frac{1}{2}\right)$ represents the Poisson coefficient. This system describes the transversal displacement $u=u(x, y, t)$ of a thin vibrating plate subjected to internal time-dependent frictional damping and boundary viscoelastic damping.

The uniform stabilization of Kirchhoff plates with linear or nonlinear internal feedback, with $\theta \equiv 1$, was investigated by several authors. In Ammari and Tucsnak [4], Cavalcanti et al. [7], Guzman and Tucsnak [11], Komornik [18], Pazoto et al. [39], and Vasconcellos and Teixeira [41], it was proved that if $h$ satisfies

$$
c_{1} \min \left\{|s|,|s|^{q}\right\} \leqslant|h(s)| \leqslant c_{2} \max \left\{|s|,|s|^{\frac{1}{q}}\right\},
$$

where $c_{1}, c_{2}$ are positive constants, then for $q=1$ the energy decay rate is exponential while for $q>1$ we obtain a polynomial decay rate. Similar results were also obtained for boundary frictional damping (see Horn [14], Komornik [19], Lagnese [21], Lasiecka [22], and Ji and Lasiecka [15]). Decay results for arbitrary growth of the frictional damping term have been given by Amroun and Benaissa [5] motivated by the works done by Lasiecka and Tataru [22], Liu and Zuazua [24], and Martinez [26,27] for damped wave equations. They established an explicit formula for the energy decay rates that need not to be of exponential or polynomial types. Similarly, Han and Wang [12] studied a coupled system of plate and wave equations and used internal frictional damping terms without imposing growth conditions near zero to achieve the stability and controllability of the system. In the presence of the time dependent coefficient $\theta(t)$, Mustafa [35] and Mustafa and Messaoudi [36] established for the wave equation a general energy decay result depending on both $h$ and $\theta$.

On the other hand, when the unique damping mechanism is given by memory conditions, we refer to Lagnese [20] and Rivera et al. [32] who considered internal viscoelastic damping and proved that the energy decays exponentially if the relaxation function $g$ decays exponentially and polynomially if $g$ decays polynomially. The same results were obtained by Alabau-Boussouira et al. [3] for a more general abstract equation. For boundary viscoelastic damping, if $k_{i}$ is the resolvent kernel of $\frac{-g_{i}^{\prime}}{g_{i}(0)}$ for $i=1,2$, Santos and Junior [40] showed that the energy decays exponentially (polynomially), provided the resolvent kernels also decay exponentially (polynomially). In Rivera et al. [33,34] investigated a class of abstract viscoelastic systems of the form

$$
u_{t t}(t)+\mathcal{A} u(t)-\left(g * A^{\beta} u\right)(t)=0,
$$

where $A$ is a strictly positive, self-adjoint operator with $D(A)$ a subset of a Hilbert space and $*$ denotes the convolution product in the variable $t$. The authors showed that solutions for (1.6), when $0<\beta<1$, decay polynomially even if the kernel $g$ decays exponentially. While, in the case $\beta=1$, the solution energy decays at the same decay rate as the relaxation function.

Then, a natural question was raised: how does the energy behave as the kernel function does not necessarily decay polynomially or exponentially? Han and Wang gave an answer to the above question when treating (1.6), for $\beta=1$, in [13]. They considered relaxation functions satisfying

$$
g^{\prime}(t) \leqslant-\xi(t) g(t), \quad \forall t \geqslant 0,
$$


where $\xi: \mathbb{R}_{+} \rightarrow \mathbb{R}_{+}$is a nonincreasing differentiable function with

$$
\left|\frac{\xi^{\prime}(t)}{\xi(t)}\right| \leqslant k, \quad \forall t \geqslant 0
$$

for some constant $k$ and showed that the rate of the decay of the energy is exactly the rate of decay of $g$ which is not necessarily of polynomial or exponential decay type. These conditions (1.7) and (1.8) on $g$ where first used by Messaoudi $[28,29]$ in studying a viscoelastic wave equation. After that, Messaoudi and Mustafa [30] and Mustafa and Messaoudi [37] eliminated condition (1.8) and used only (1.7) to establish more general stability results of viscoelastic Timoshenko beams. Similarly, condition (1.7) was used by Ferreira and Messaoudi [9] to treat a nonlinear viscoelastic plate equation with a $\vec{p}(x, t)$ Laplacian operator. We also mention the work of Alabau-Boussouira and Cannarsa [2] who considered wave equation with memory whose relaxation function is satisfying

$$
g^{\prime} \leqslant-\chi(g(t))
$$

where $\chi$ is a non-negative function, with $\chi(0)=\chi^{\prime}(0)=0$, and $\chi$ is strictly increasing and strictly convex on $\left(0, k_{0}\right]$, for some $k_{0}>0$. They also required that

$$
\int_{0}^{k_{0}} \frac{d x}{\chi(x)}=+\infty, \quad \int_{0}^{k_{0}} \frac{x d x}{\chi(x)}<1, \quad \lim _{s \rightarrow 0^{+}} \inf \frac{\chi(s) / s}{\chi^{\prime}(s)}>\frac{1}{2}
$$

and proved an energy decay result. In addition to these assumptions, if

$$
\lim _{s \rightarrow 0^{+}} \sup \frac{\chi(s) / s}{\chi^{\prime}(s)}<1 \quad \text { and } \quad g^{\prime}=-\chi(g(t))
$$

then, in this case, an explicit rate of decay is given. Recently, the above conditions were strongly weakened by Mustafa and Messaoudi [38] and an explicit and general decay rate formula was obtained.

The interaction between viscoelastic and frictional dampings was considered by several authors. Cavalcanti and Oquendo [8] looked into wave equation of the form

$$
u_{t t}-\Delta u+\int_{0}^{t} \operatorname{div}[a(x) g(t-s) \nabla u(s)] d s+b(x) h\left(u_{t}\right)+f(u)=0, \quad x \in \Omega, t>0
$$

and established exponential stability for $g$ decaying exponentially and $h$ linear and polynomial stability for $g$ decaying polynomially and $h$ having a polynomial growth near zero. Using (1.7), $h$ having no restrictive growth assumption near the origin, with time dependent coefficient and $a(x)=b(x)=1$, Liu [25] proved a more general decay result. Similarly, Guesmia and Messaoudi [10] studied Timoshenko systems with frictional versus viscoelastic damping and Messaoudi and Mustafa [31] studied viscoelastic wave equation with boundary feedback and obtained general energy decay estimates. Once again, Kang $[16,17]$ imposed the condition $(1.7)$ on the relaxation functions for viscoelastic dampings in plate models which are also subject to frictional damping and they established general stability results.

Our aim in this work is to investigate (1.1)-(1.5) with both weak frictional damping and boundary viscoelastic damping. We obtain a general relation between the decay rate for the energy (when $t$ goes to infinity) and the functions $g_{i}, \theta$, and $h$ using resolvent kernels of general-type decay and without 
imposing any growth assumption near the origin on $h$. The result of this paper generalizes previous related results where it allows a larger class of functions $g$ and $h$, from which the energy decay rates are not necessarily of exponential or polynomial types and takes into account the effect of a time dependent coefficient $\theta(t)$. The proof is based on the multiplier method and makes use of some properties of convex functions including the use of the general Young's inequality and Jensen's inequality. These convexity arguments were introduced by Lasiecka and Tataru [23] and used by Liu and Zuazua [24] and AlabauBoussouira [1]. The paper is organized as follows. In Section 2, we present some notation and material needed for our work. Some technical lemmas and the proof of our main result will be given in Section 3 .

\section{Preliminaries}

We use the standard Lebesgue and Sobolev spaces with their usual scalar products and norms. Throughout this paper, $c$ is used to denote a generic positive constant. We first consider the following hypothesis

(A1). $\Omega$ is a bounded domain of $\mathbb{R}^{2}$ with a smooth boundary $\partial \Omega=\Gamma_{0} \cup \Gamma_{1}$, where $\Gamma_{0}$ and $\Gamma_{1}$ are closed and disjoint, with meas $\left(\Gamma_{0}\right)>0, v=\left(v_{1}, v_{2}\right)$ is the unit outward normal to $\partial \Omega, \eta=\left(-v_{2}, v_{1}\right)$ is the unit tangent positively oriented on $\partial \Omega$, and there exists a fixed point $x_{0} \in \mathbb{R}^{2}$ such that, for $m(x)=x-x_{0}$, $m \cdot v \leqslant 0$ on $\Gamma_{0}$ and $m \cdot v>0$ on $\Gamma_{1}$.

Remark. Hypothesis (A1) implies that there exist constants $\delta_{0}$ and $R$ such that

$$
m \cdot v \geqslant \delta_{0}>0 \quad \text { on } \Gamma_{1} \quad \text { and } \quad|m(x)| \leqslant R \quad \text { for all } x \in \Omega .
$$

We denote by $k_{i}$ the resolvent kernel of $\left(-g_{i}^{\prime} / g_{i}(0)\right)$ which satisfies

$$
k_{i}(t)+\frac{1}{g_{i}(0)}\left(g_{i}^{\prime} * k_{i}\right)(t)=-\frac{1}{g_{i}(0)} g_{i}^{\prime}(t), \quad \forall i=1,2,
$$

where $*$ points to the convolution product

$$
(u * v)(t)=\int_{0}^{t} u(t-s) v(s) d s .
$$

By differentiating Eqs (1.3) and (1.4), we arrive at the following Volterra equations:

$$
\begin{aligned}
& \beta_{2} u+\frac{1}{g_{1}(0)} g_{1}^{\prime} * \beta_{2} u=\frac{1}{g_{1}(0)} u_{t}, \\
& \beta_{1} u+\frac{1}{g_{2}(0)} g_{2}^{\prime} * \beta_{1} u=-\frac{1}{g_{2}(0)} \frac{\partial u_{t}}{\partial v} .
\end{aligned}
$$

Using the Volterra's inverse operator and taking $\tau_{i}=\frac{1}{g_{i}(0)}$, for $i=1$, 2, we get

$$
\begin{aligned}
& \beta_{2} u=\tau_{1}\left\{u_{t}+k_{1} * u_{t}\right\}, \quad \text { on } \Gamma_{1} \times(0, \infty), \\
& \beta_{1} u=-\tau_{2}\left\{\frac{\partial u_{t}}{\partial v}+k_{2} * \frac{\partial u_{t}}{\partial v}\right\}, \quad \text { on } \Gamma_{1} \times(0, \infty),
\end{aligned}
$$


which gives, assuming throughout the paper that $u_{0} \equiv 0$,

$$
\begin{aligned}
& \beta_{2} u=\tau_{1}\left\{u_{t}+k_{1}(0) u+k_{1}^{\prime} * u\right\}, \quad \text { on } \Gamma_{1} \times(0, \infty), \\
& \beta_{1} u=-\tau_{2}\left\{\frac{\partial u_{t}}{\partial v}+k_{2}(0) \frac{\partial u}{\partial v}+k_{2}^{\prime} * \frac{\partial u}{\partial v}\right\}, \quad \text { on } \Gamma_{1} \times(0, \infty) .
\end{aligned}
$$

Therefore, we use (2.1) and (2.2) instead of the boundary conditions (1.3) and (1.4), and also consider the following assumptions.

(A2). $k_{i}: \mathbb{R}_{+} \rightarrow \mathbb{R}_{+}$, for $i=1,2$, are $C^{2}$ functions such that

$$
k_{i}(0)>0, \quad \lim _{t \rightarrow \infty} k_{i}(t)=0, \quad k_{i}^{\prime}(t) \leqslant 0
$$

and there exists a positive function $H \in C^{1}\left(\mathbb{R}_{+}\right)$and $H$ is a linear function or it is a strictly increasing and strictly convex $C^{2}$ function on $(0, r], r<1$, with $H(0)=H^{\prime}(0)=0$, such that

$$
k_{i}^{\prime \prime}(t) \geqslant H\left(-k_{i}^{\prime}(t)\right), \quad(i=1,2) \forall t>0 .
$$

(A3). $h: \mathbb{R} \rightarrow \mathbb{R}$ is a nondecreasing $C^{0}$ function and there exist constants $c_{1}, c_{2}>0$ such that

$$
\begin{aligned}
& c_{1}|s| \leqslant|h(s)| \leqslant c_{2}|s| \quad \text { if }|s| \geqslant r, \\
& s^{2}+h^{2}(s) \leqslant H^{-1}(\operatorname{sh}(s)) \quad \text { if }|s| \leqslant r .
\end{aligned}
$$

(A4). $\theta: \mathbb{R}_{+} \rightarrow \mathbb{R}_{+}$is a nonincreasing $C^{1}$ function.

In the sequel we assume that system (1.1)-(1.5) has a unique solution

$$
u \in L^{\infty}\left(\mathbb{R}_{+} ; H^{4}(\Omega) \cap W\right) \cap W^{1, \infty}\left(\mathbb{R}_{+} ; W\right) \cap W^{2, \infty}\left(\mathbb{R}_{+} ; L^{2}(\Omega)\right),
$$

where $W=\left\{w \in H^{2}(\Omega): w=\frac{\partial w}{\partial v}=0\right.$ on $\left.\Gamma_{0}\right\}$. This result can be proved, for initial data in suitable function spaces, using standard arguments such as the Galerkin method (see [40]).

Let us define the bilinear form $a(\cdot, \cdot)$ as follows

$$
a(u, v)=\int_{\Omega}\left\{u_{x x} v_{x x}+u_{y y} v_{y y}+\mu\left(u_{x x} v_{y y}+u_{y y} v_{x x}\right)+2(1-\mu) u_{x y} v_{x y}\right\} d x d y
$$

and, as meas $\Gamma_{0}>0$, we know that $\sqrt{a(u, u)}$ is an equivalent norm on $W$; that is, for some positive constants $\alpha$ and $\beta$,

$$
\alpha\|u\|_{H^{2}(\Omega)}^{2} \leqslant a(u, u) \leqslant \beta\|u\|_{H^{2}(\Omega)}^{2} .
$$

We state the following lemma which will be useful in what follows. 
Lemma 2.1 ([21]). Let $u$ and $v$ be functions in $H^{4}(\Omega)$ and $\mu \in \mathbb{R}$. Then we have

$$
\int_{\Omega}\left(\Delta^{2} u\right) v d x=a(u, v)+\int_{\Gamma}\left\{\left(\beta_{2} u\right) v-\left(\beta_{1} u\right) \frac{\partial v}{\partial v}\right\} d \Gamma
$$

and

$$
\begin{aligned}
\int_{\Omega}(m \cdot \nabla v) \Delta^{2} v d x= & a(v, v)+\frac{1}{2} \int_{\Gamma} m \cdot v\left[v_{x x}^{2}+v_{y y}^{2}+2 \mu v_{x x} v_{y y}+2(1-\mu) v_{x y}^{2}\right] d \Gamma \\
& +\int_{\Gamma}\left[\left(\beta_{2} v\right) m \cdot \nabla v-\left(\beta_{1} v\right) \frac{\partial}{\partial v}(m \cdot \nabla v)\right] d \Gamma .
\end{aligned}
$$

Now, we introduce the energy functional

$$
\begin{aligned}
E(t):= & \frac{1}{2}\left[\int_{\Omega}\left|u_{t}\right|^{2} d x+a(u, u)+\tau_{1} \int_{\Gamma_{1}}\left(k_{1}(t)|u|^{2}-\left(k_{1}^{\prime} \circ u\right)\right) d \Gamma\right. \\
& \left.+\tau_{2} \int_{\Gamma_{1}}\left(k_{2}(t)\left|\frac{\partial u}{\partial v}\right|^{2}-\left(k_{2}^{\prime} \circ \frac{\partial u}{\partial v}\right)\right) d \Gamma\right],
\end{aligned}
$$

where

$$
(f \circ w)(t)=\int_{0}^{t} f(t-s)|w(t)-w(s)|^{2} d s .
$$

Our main stability result is the following

Theorem 2.1. Assume that (A1)-(A4) hold. Then there exist positive constants $c_{1}, c_{2}, c_{3}$ and $\varepsilon_{0}$ such that the solution of (1.1)-(1.5) satisfies

$$
E(t) \leqslant c_{3} H_{1}^{-1}\left(c_{1} \int_{0}^{t} \theta(s) d s+c_{2}\right) \quad \forall t \geqslant 0
$$

where

$$
H_{1}(t)=\int_{t}^{1} \frac{1}{s H_{0}^{\prime}\left(\varepsilon_{0} s\right)} d s \quad \text { and } \quad H_{0}(t)=H(D(t))
$$

provided that $D$ is a positive $C^{1}$ function, with $D(0)=0$, for which $H_{0}$ is a strictly increasing and strictly convex $C^{2}$ function on $(0, r]$ and

$$
\int_{0}^{+\infty} \frac{-k_{i}^{\prime}(s)}{H_{0}^{-1}\left(k_{i}^{\prime \prime}(s)\right)} d s<+\infty \quad \text { for } i=1,2 .
$$

Moreover, if $\int_{0}^{1} H_{1}(t) d t<+\infty$ for some choice of $D$, then we have the improved estimate

$$
E(t) \leqslant c_{3} G^{-1}\left(c_{1} \int_{0}^{t} \theta(s) d s+c_{2}\right) \quad \text { where } G(t)=\int_{t}^{1} \frac{1}{s H^{\prime}\left(\varepsilon_{0} s\right)} d s .
$$


In particular, this last estimate is valid for the special case $H(t)=c t^{p}$, for $1 \leqslant p<\frac{3}{2}$.

\section{Remarks.}

1. Using the properties of $H$, one can show that the function $H_{1}$ is strictly decreasing and convex on $(0,1]$, with $\lim _{t \rightarrow 0} H_{1}(t)=+\infty$. Therefore, Theorem 2.1 ensures

$$
\lim _{t \rightarrow+\infty} E(t)=0
$$

2. Hypothesis (A3) implies that $\operatorname{sh}(s)>0$, for all $s \neq 0$.

3. The condition (A3), with $r=1$ and $\theta \equiv 1$, was introduced and employed by Lasiecka and Tataru [23] in their study of the asymptotic behavior of solutions of nonlinear wave equations with nonlinear frictional boundary damping where they obtained decay estimates that depend on the solution of an explicit nonlinear ordinary differential equation. It was also shown there that the monotonicity and continuity of $h$ guarantee the existence of the function $H$ with the properties stated in (A3). In our present work, we study the plate equation with both frictional damping, modulated by a time dependent coefficient $\theta(t)$, and boundary viscoelastic damping. We investigate the influence of these simultaneous damping mechanisms on the decay rate of the energy and establish an explicit and general energy decay formula, depending on the resolvent kernels $k_{1}$ and $k_{2}, h$, and $\theta$.

4. The usual exponential and polynomial decay rate estimates, already proved for $H(t)=c t^{p}, 1 \leqslant$ $p<3 / 2$, are special cases of our result. We will provide a "simpler" proof for these special cases.

5. The condition $k_{i}^{\prime \prime} \geqslant d\left(-k_{i}^{\prime}\right)^{p}, 1 \leqslant p<3 / 2$, assumes $\left(-k_{i}^{\prime}(t)\right) \leqslant \omega e^{-d t}$ when $p=1$ and $\left(-k_{i}^{\prime}(t)\right) \leqslant \frac{\omega}{t^{\frac{1}{p-1}}}$ when $1<p<3 / 2$. Our result allows resolvent kernels whose derivatives are not necessarily of exponential or polynomial decay. For instance, if

$$
k_{i}^{\prime}(t)=-\exp \left(-t^{q}\right), \quad i=1,2
$$

for $0<q<1$, then $k_{i}^{\prime \prime}(t)=H\left(-k_{i}^{\prime}(t)\right)$ where, for $t \in(0, r], r<1$,

$$
H(t)=\frac{q t}{[\ln (1 / t)]^{\frac{1}{q}-1}}
$$

which satisfies hypothesis (A2). Also, by taking $D(t)=t^{\alpha},(2.8)$ is satisfied for any $\alpha>1$. Therefore, if $h$ satisfies (A3) with this function $H$, then we can use Theorem 2.1 and do some calculations (see [38]) to deduce that the energy decays at the rate

$$
E(t) \leqslant c \exp \left(-\omega\left[\int_{0}^{t} \theta(s) d s\right]^{q}\right)
$$

6. The well-known Jensen's inequality will be of essential use in establishing our main result. If $F$ is a convex function on $[a, b], f: \Omega \rightarrow[a, b]$ and $j$ are integrable functions on $\Omega, j(x) \geqslant 0$, and $\int_{\Omega} j(x) d x=C>0$, then Jensen's inequality states that

$$
F\left[\frac{1}{C} \int_{\Omega} f(x) j(x) d x\right] \leqslant \frac{1}{C} \int_{\Omega} F[f(x)] j(x) d x .
$$


7. Since $\lim _{t \rightarrow+\infty} k_{i}(t)=0$ and $\left(-k_{i}^{\prime}(t)\right)$ is nonnegative and nonincreasing, then we can easily deduce that $\lim _{t \rightarrow+\infty}\left(-k_{i}^{\prime}(t)\right)=0$. Similarly, assuming the existence of the limit, we find that $\lim _{t \rightarrow+\infty} k_{i}^{\prime \prime}(t)=0$. Hence, there is $t_{1}>0$ large enough such that $k_{i}^{\prime}\left(t_{1}\right)<0$ and

$$
\max \left\{k_{i}(t),-k_{i}^{\prime}(t), k_{i}^{\prime \prime}(t)\right\}<\min \left\{r, H(r), H_{0}(r)\right\}, \quad(i=1,2) \forall t \geqslant t_{1} .
$$

As $k_{i}^{\prime}$ is nondecreasing, $k_{i}^{\prime}(0)<0$ and $k_{i}^{\prime}\left(t_{1}\right)<0$, then $k_{i}^{\prime}(t)<0$ for any $t \in\left[0, t_{1}\right]$ and

$$
0<-k_{i}^{\prime}\left(t_{1}\right) \leqslant-k_{i}^{\prime}(t) \leqslant-k_{i}^{\prime}(0), \quad(i=1,2) \forall t \in\left[0, t_{1}\right]
$$

Therefore, since $H$ is a positive continuous function, then

$$
a \leqslant H\left(-k_{i}^{\prime}(t)\right) \leqslant b, \quad(i=1,2) \forall t \in\left[0, t_{1}\right]
$$

for some positive constants $a$ and $b$. Consequently, for all $t \in\left[0, t_{1}\right]$,

$$
k_{i}^{\prime \prime}(t) \geqslant H\left(-k_{i}^{\prime}(t)\right) \geqslant a=\frac{a}{k_{i}^{\prime}(0)} k_{i}^{\prime}(0) \geqslant \frac{a}{k_{i}^{\prime}(0)} k_{i}^{\prime}(t) \quad(i=1,2)
$$

which gives, for some positive constant $d$,

$$
k_{i}^{\prime \prime}(t) \geqslant-d k_{i}^{\prime}(t), \quad(i=1,2) \forall t \in\left[0, t_{1}\right]
$$

8. If different functions $H_{1}, H_{2}$, and $H_{3}$ have the properties mentioned in (A2) and (A3) such that $k_{1}^{\prime \prime}(t) \geqslant H_{1}\left(-k_{1}^{\prime}(t)\right), k_{2}^{\prime \prime}(t) \geqslant H_{2}\left(-k_{2}^{\prime}(t)\right)$, and $s^{2}+h^{2}(s) \leqslant H_{3}^{-1}(\operatorname{sh}(s))$, then there is $r<$ $\min \left\{r_{1}, r_{2}, r_{3}\right\}$ small enough so that, say, $H_{1}(t) \leqslant \min \left\{H_{2}(t), H_{3}(t)\right\}$ on the interval $(0, r]$. Thus, the function $H(t)=H_{1}(t)$ satisfies both (A2) and (A3), $\forall t \geqslant t_{1}$.

\section{Proof of the main result}

In this section we prove Theorem 2.1. For this purpose, we establish several lemmas.

Lemma 3.1. Under the assumptions (A1)-(A4), the energy functional satisfies, along the solution of (1.1), the estimate

$$
\begin{aligned}
E^{\prime}(t)= & -\theta(t) \int_{\Omega} u_{t} h\left(u_{t}\right) d x-\frac{\tau_{1}}{2} \int_{\Gamma_{1}}\left(2\left|u_{t}\right|^{2}-k_{1}^{\prime}(t)|u|^{2}+k_{1}^{\prime \prime} \circ u\right) d \Gamma \\
& -\frac{\tau_{2}}{2} \int_{\Gamma_{1}}\left(2\left|\frac{\partial u_{t}}{\partial v}\right|^{2}-k_{2}^{\prime}(t)\left|\frac{\partial u}{\partial v}\right|^{2}+k_{2}^{\prime \prime} \circ \frac{\partial u}{\partial v}\right) d \Gamma \\
\leqslant & 0 .
\end{aligned}
$$


Proof. Multiplying the equation (1.1) by $u_{t}$, integrating by parts over $\Omega$, and using (2.5) and the boundary conditions (2.1) and (2.2), we get

$$
\begin{aligned}
& \frac{1}{2} \frac{d}{d t}\left\{\int_{\Omega}\left|u_{t}\right|^{2} d x+a(u, u)\right\} \\
&=-\int_{\Gamma_{1}}\left(\beta_{2} u\right) u_{t} d \Gamma+\int_{\Gamma_{1}}\left(\beta_{1} u\right) \frac{\partial u_{t}}{\partial v} d \Gamma-\theta(t) \int_{\Omega} u_{t} h\left(u_{t}\right) d x \\
&=-\tau_{1} \int_{\Gamma_{1}} u_{t}^{2}-\frac{k_{1}(0) \tau_{1}}{2} \frac{d}{d t} \int_{\Gamma_{1}} u^{2}-\tau_{1} \int_{\Gamma_{1}}\left\{k_{1}^{\prime} * u\right\} u_{t} d \Gamma-\tau_{2} \int_{\Gamma_{1}}\left|\frac{\partial u_{t}}{\partial v}\right|^{2} \\
&-\frac{k_{2}(0) \tau_{2}}{2} \frac{d}{d t} \int_{\Gamma_{1}}\left|\frac{\partial u}{\partial v}\right|^{2}-\tau_{2} \int_{\Gamma_{1}}\left\{k_{2}^{\prime} * \frac{\partial u}{\partial v}\right\} \frac{\partial u_{t}}{\partial v} d \Gamma-\theta(t) \int_{\Omega} u_{t} h\left(u_{t}\right) d x
\end{aligned}
$$

Then, making use of the identity

$$
(f * w) w_{t}=-\frac{1}{2} f(t)|w(t)|^{2}+\frac{1}{2} f^{\prime} \circ w-\frac{1}{2} \frac{d}{d t}\left[f \circ w-\left(\int_{0}^{t} f(s) d s\right)|w|^{2}\right],
$$

our conclusion follows.

Now we are going to construct a Lyapunov functional $\mathcal{L}$ equivalent to $E$, with which we can show the desired result.

Lemma 3.2. Under the assumptions (A1)-(A4), the functional

$$
\psi(t):=\int_{\Omega}(m \cdot \nabla u) u_{t} d x
$$

satisfies, along the solution, the estimate

$$
\begin{aligned}
\frac{d}{d t} \psi(t) \leqslant & \frac{1}{2} \int_{\Gamma 1} m \cdot v\left|u_{t}\right|^{2} d \Gamma-\int_{\Omega}\left|u_{t}\right|^{2} d x-\left(1-\frac{\epsilon c}{2}\right) a(u, u) \\
& +\frac{2 \tau_{1}^{2}}{\epsilon} \int_{\Gamma_{1}}\left[\left|u_{t}\right|^{2}+k_{1}^{2}(t)|u|^{2}-k_{1}(0)\left(k_{1}^{\prime} \circ u\right)\right] d \Gamma+\frac{c}{2 \epsilon} \int_{\Omega} h^{2}\left(u_{t}\right) d x \\
& +\frac{2 \tau_{2}^{2}}{\epsilon} \int_{\Gamma_{1}}\left[\left|\frac{\partial u_{t}}{\partial v}\right|^{2}+k_{2}^{2}(t)\left|\frac{\partial u}{\partial v}\right|^{2}-k_{2}(0)\left(k_{2}^{\prime} \circ \frac{\partial u}{\partial v}\right)\right] d \Gamma \\
& -\left(\frac{1}{2}-\frac{\epsilon c}{2}\right) \int_{\Gamma_{1}} m \cdot v\left[u_{x x}^{2}+u_{y y}^{2}+2 \mu u_{x x} u_{y y}+2(1-\mu) u_{x y}^{2}\right] d \Gamma .
\end{aligned}
$$

Proof. Direct computations, taking $v=u$ in (2.6), we get

$$
\frac{d}{d t} \psi(t)=\int_{\Omega}\left(m \cdot \nabla u_{t}\right) u_{t} d x+\int_{\Omega}(m \cdot \nabla u) u_{t t} d x
$$




$$
\begin{aligned}
= & \frac{1}{2} \int_{\Gamma 1} m \cdot v\left|u_{t}\right|^{2} d \Gamma-\int_{\Omega}\left|u_{t}\right|^{2} d x-\theta(t) \int_{\Omega}(m \cdot \nabla u) h\left(u_{t}\right) d x \\
& -a(u, u)-\int_{\Gamma}\left[\left(\beta_{2} u\right)(m \cdot \nabla u)-\left(\beta_{1} u\right) \frac{\partial}{\partial v}(m \cdot \nabla u)\right] d \Gamma \\
& -\frac{1}{2} \int_{\Gamma} m \cdot v\left[u_{x x}^{2}+u_{y y}^{2}+2 \mu u_{x x} u_{y y}+2(1-\mu) u_{x y}^{2}\right] d \Gamma .
\end{aligned}
$$

Let us examine the integrals over $\Gamma_{0}$ in (3.3). Since $u=\frac{\partial u}{\partial v}=0$ on $\Gamma_{0}$, we have $B_{1} u=B_{2} u=0$ on $\Gamma_{0}$ and

$$
\begin{aligned}
& \frac{\partial}{\partial v}(m \cdot \nabla u)=(m \cdot v) \Delta u \\
& u_{x x}^{2}+u_{y y}^{2}+2 \mu u_{x x} u_{y y}+2(1-\mu) u_{x y}^{2}=(\Delta u)^{2} \quad \text { on } \Gamma_{0}
\end{aligned}
$$

since

$$
u_{x x} u_{y y}-\left(u_{x y}\right)^{2}=0 \quad \text { on } \Gamma_{0} .
$$

Therefore, from (3.3), we have

$$
\begin{aligned}
\frac{d}{d t} \psi(t)= & \frac{1}{2} \int_{\Gamma_{1}} m \cdot v\left|u_{t}\right|^{2} d \Gamma-\int_{\Omega}\left|u_{t}\right|^{2} d x-\theta(t) \int_{\Omega}(m \cdot \nabla u) h\left(u_{t}\right) d x \\
& -a(u, u)+\frac{1}{2} \int_{\Gamma_{0}} m \cdot v(\Delta u)^{2} d \Gamma \\
& -\frac{1}{2} \int_{\Gamma_{1}} m \cdot v\left[u_{x x}^{2}+u_{y y}^{2}+2 \mu u_{x x} u_{y y}+2(1-\mu) u_{x y}^{2}\right] d \Gamma \\
& -\int_{\Gamma_{1}}\left(\beta_{2} u\right)(m \cdot \nabla u) d \Gamma+\int_{\Gamma 1}\left(\beta_{1} u\right) \frac{\partial}{\partial v}(m \cdot \nabla u) d \Gamma .
\end{aligned}
$$

Using the Young inequality, we have

$$
\begin{aligned}
& \left|\int_{\Gamma_{1}}\left(\beta_{2} u\right)(m \cdot \nabla u) d \Gamma\right| \leqslant \frac{1}{2 \epsilon} \int_{\Gamma_{1}}\left|\beta_{2} u\right|^{2} d \Gamma+\frac{\epsilon}{2} \int_{\Gamma_{1}}|m \cdot \nabla u|^{2} d \Gamma, \\
& \left|\int_{\Gamma 1}\left(\beta_{1} u\right) \frac{\partial}{\partial v}(m \cdot \nabla u) d \Gamma\right| \leqslant \frac{1}{2 \epsilon} \int_{\Gamma_{1}}\left|\beta_{1} u\right|^{2} d \Gamma+\frac{\epsilon}{2} \int_{\Gamma_{1}}\left|\frac{\partial}{\partial v}(m \cdot \nabla u)\right|^{2} d \Gamma, \\
& -\theta(t) \int_{\Omega}(m \cdot \nabla u) h\left(u_{t}\right) d x \leqslant \frac{c}{2 \epsilon} \int_{\Omega} h^{2}\left(u_{t}\right) d x+\frac{\epsilon}{2} \int_{\Omega}|m \cdot \nabla u|^{2} d x,
\end{aligned}
$$

where $\epsilon$ is a positive constant. Using the trace theory, we obtain

$$
\int_{\Gamma_{1}}|m \cdot \nabla u|^{2} d \Gamma+\int_{\Gamma_{1}}\left|\frac{\partial}{\partial v}(m \cdot \nabla u)\right|^{2} d \Gamma+\int_{\Omega}|m \cdot \nabla u|^{2} d x
$$




$$
\leqslant c a(u, u)+c \int_{\Gamma_{1}} m \cdot v\left[u_{x x}^{2}+u_{y y}^{2}+2 \mu u_{x x} u_{y y}+2(1-\mu) u_{x y}^{2}\right] d \Gamma .
$$

Substituting the inequalities (3.5)-(3.8) into (3.4) and taking into account the fact that $m \cdot v \leqslant 0$ on $\Gamma_{0}$, we have

$$
\begin{aligned}
\frac{d}{d t} \psi(t) \leqslant & \frac{1}{2} \int_{\Gamma 1} m \cdot v\left|u_{t}\right|^{2} d \Gamma-\int_{\Omega}\left|u_{t}\right|^{2} d x-\left(1-\frac{\epsilon c}{2}\right) a(u, u) \\
& +\frac{1}{2 \epsilon} \int_{\Gamma_{1}}\left|\beta_{1} u\right|^{2} d \Gamma+\frac{1}{2 \epsilon} \int_{\Gamma_{1}}\left|\beta_{2} u\right|^{2} d \Gamma+\frac{c}{2 \epsilon} \int_{\Omega} h^{2}\left(u_{t}\right) d x \\
& -\left(\frac{1}{2}-\frac{\epsilon c}{2}\right) \int_{\Gamma_{1}} m \cdot v\left[u_{x x}^{2}+u_{y y}^{2}+2 \mu u_{x x} u_{y y}+2(1-\mu) u_{x y}^{2}\right] d \Gamma .
\end{aligned}
$$

Since, by Hölder inequality,

$$
\begin{aligned}
\left(k_{1}^{\prime} * u\right)(t) & =-\int_{0}^{t} k_{1}^{\prime}(t-s)(u(t)-u(s)) d s+k_{1}(t) u(t)-k_{1}(0) u(t) \\
& \leqslant\left[-\int_{0}^{t} k_{1}^{\prime}(s) d s\right]^{\frac{1}{2}}\left[\left(k_{1}^{\prime} \circ u\right)(t)\right]^{\frac{1}{2}}+k_{1}(t) u(t)-k_{1}(0) u(t) \\
& \leqslant\left[-k_{1}(0)\left(k_{1}^{\prime} \circ u\right)(t)\right]^{\frac{1}{2}}+k_{1}(t) u(t)-k_{1}(0) u(t)
\end{aligned}
$$

then

$$
\beta_{2} u \leqslant \tau_{1}\left\{u_{t}+k_{1}(t) u+\left[-k_{1}(0)\left(k_{1}^{\prime} \circ u\right)(t)\right]^{\frac{1}{2}}\right\},
$$

similarly

$$
\beta_{1} u \leqslant-\tau_{2}\left\{\frac{\partial u_{t}}{\partial v}+k_{2}(t) \frac{\partial u}{\partial v}+\left[-k_{2}(0)\left(k_{2}^{\prime} \circ \frac{\partial u}{\partial v}\right)(t)\right]^{\frac{1}{2}}\right\} .
$$

Consequently, our conclusion easily follows.

Proof of Theorem 2.1. For $N>0$, we define

$$
\mathcal{L}(t):=N E(t)+\psi(t) .
$$

Combining (3.1) and (3.2) and using the facts that $k_{i}^{\prime}<0, k_{i}^{\prime \prime}>0$ and $|m \cdot v| \leqslant R$, we obtain

$$
\begin{aligned}
\mathcal{L}^{\prime}(t) \leqslant & -\left(\tau_{1} N-\frac{R}{2}-\frac{2 \tau_{1}^{2}}{\epsilon}\right) \int_{\Gamma_{1}}\left|u_{t}\right|^{2} d \Gamma-\left(\tau_{2} N-\frac{2 \tau_{2}^{2}}{\epsilon}\right) \int_{\Gamma_{1}}\left|\frac{\partial u_{t}}{\partial v}\right|^{2} d \Gamma \\
& -\left(1-\frac{\epsilon c}{2}\right) a(u, u)+\frac{2 \tau_{1}^{2}}{\epsilon} \int_{\Gamma_{1}} k_{1}^{2}(t)|u|^{2} d \Gamma+\frac{2 \tau_{2}^{2}}{\epsilon} \int_{\Gamma_{1}} k_{2}^{2}(t)\left|\frac{\partial u}{\partial v}\right|^{2} d \Gamma
\end{aligned}
$$




$$
\begin{aligned}
& -\int_{\Omega}\left|u_{t}\right|^{2} d x-\frac{2 \tau_{1}^{2} k_{1}(0)}{\epsilon} \int_{\Gamma_{1}} k_{1}^{\prime} \circ u d \Gamma-\frac{2 \tau_{2}^{2} k_{2}(0)}{\epsilon} \int_{\Gamma_{1}} k_{2}^{\prime} \circ \frac{\partial u}{\partial v} d \Gamma \\
& -\left(\frac{1}{2}-\frac{\epsilon c}{2}\right) \int_{\Gamma_{1}} m \cdot v\left[u_{x x}^{2}+u_{y y}^{2}+2 \mu u_{x x} u_{y y}+2(1-\mu) u_{x y}^{2}\right] d \Gamma \\
& +\frac{c}{2 \epsilon} \int_{\Omega} h^{2}\left(u_{t}\right) d x .
\end{aligned}
$$

Then choosing $0<\epsilon<\frac{1}{c}$, and $N$ large enough so that

$$
\tau_{1} N-\frac{R}{2}-\frac{2 \tau_{1}^{2}}{\epsilon}>0, \quad \tau_{2} N-\frac{2 \tau_{2}^{2}}{\epsilon}>0,
$$

so, we arrive at

$$
\begin{aligned}
\mathcal{L}^{\prime}(t) \leqslant & -\int_{\Omega}\left|u_{t}\right|^{2} d x-\frac{1}{2} a(u, u)+c \int_{\Omega} h^{2}\left(u_{t}\right) d x+\frac{2 \iota_{1}^{2}}{\epsilon} \int_{\Gamma_{1}} k_{1}^{2}(t)|u|^{2} d \Gamma \\
& +\frac{2 \iota_{2}^{2}}{\epsilon} \int_{\Gamma_{1}} k_{2}^{2}(t)\left|\frac{\partial u}{\partial v}\right|^{2} d \Gamma-c \int_{\Gamma_{1}} k_{1}^{\prime} \circ u d \Gamma-c \int_{\Gamma_{1}} k_{2}^{\prime} \circ \frac{\partial u}{\partial v} d \Gamma,
\end{aligned}
$$

which, using Trace theory and the fact that $\lim _{t \rightarrow \infty} k_{i}(t)=0$, for $i=1,2$, yields, for large $t_{1}$,

$$
\mathcal{L}^{\prime}(t) \leqslant-m E(t)-c \int_{\Gamma_{1}} k_{1}^{\prime} \circ u d \Gamma-c \int_{\Gamma_{1}} k_{2}^{\prime} \circ \frac{\partial u}{\partial v} d \Gamma+c \int_{\Omega} h^{2}\left(u_{t}\right) d x, \quad \forall t \geqslant t_{1} .
$$

On the other hand, we can choose $N$ even larger (if needed) so that

$$
\mathcal{L}(t) \sim E(t),
$$

which means that, for some constants $\alpha_{1}, \alpha_{2}>0$,

$$
\alpha_{1} E(t) \leqslant \mathcal{L}(t) \leqslant \alpha_{2} E(t)
$$

Now, we consider the following partition of $\Omega$

$$
\Omega_{1}=\left\{x \in \Omega:\left|u_{t}\right| \leqslant r\right\}, \quad \Omega_{2}=\left\{x \in \Omega:\left|u_{t}\right|>r\right\}
$$

and use (A3), (A4), (2.11), and (3.1) to conclude that, for any $t \geqslant t_{1}$,

$$
\begin{aligned}
& -\theta(t) \int_{0}^{t_{1}} k_{1}^{\prime}(s) \int_{\Gamma_{1}}|u(t)-u(t-s)|^{2} d \Gamma d s+c \theta(t) \int_{\Omega_{2}} h^{2}\left(u_{t}\right) d x \\
& \leqslant \frac{c}{d} \int_{0}^{t_{1}} k_{1}^{\prime \prime}(s) \int_{\Gamma_{1}}|u(t)-u(t-s)|^{2} d \Gamma d s+c \theta(t) \int_{\Omega_{2}} u_{t} h\left(u_{t}\right) d x \\
& \quad \leqslant-c E^{\prime}(t),
\end{aligned}
$$




$$
\begin{aligned}
& -\theta(t) \int_{0}^{t_{1}} k_{2}^{\prime}(s) \int_{\Gamma_{1}}\left|\frac{\partial u(t)}{\partial v}-\frac{\partial u(t-s)}{\partial v}\right|^{2} d \Gamma d s \\
& \leqslant \frac{c}{d} \int_{0}^{t_{1}} k_{2}^{\prime \prime}(s) \int_{\Gamma_{1}}\left|\frac{\partial u(t)}{\partial v}-\frac{\partial u(t-s)}{\partial v}\right|^{2} \leqslant-c E^{\prime}(t) .
\end{aligned}
$$

Next, we take $F(t)=\theta(t) \mathcal{L}(t)+2 c E(t)$, which is clearly equivalent to $E(t)$ as $\theta$ is nonincreasing, and use (3.10) and (3.12)-(3.13), to get, for all $t \geqslant t_{1}$,

$$
\begin{aligned}
F^{\prime}(t) \leqslant & -m \theta E(t)-c \theta \int_{t_{1}}^{t} k_{1}^{\prime}(s) \int_{\Gamma_{1}}|u(t)-u(t-s)|^{2} d \Gamma d s \\
& -c \theta \int_{t_{1}}^{t} k_{2}^{\prime}(s) \int_{\Gamma_{1}}\left|\frac{\partial u(t)}{\partial v}-\frac{\partial u(t-s)}{\partial v}\right|^{2} d \Gamma d s+c \theta \int_{\Omega_{1}} h^{2}\left(u_{t}\right) d x .
\end{aligned}
$$

(I) $H(t)=c t^{p}$ and $1 \leqslant p<\frac{3}{2}$ : This means, using Holder's inequality, that

$$
\begin{aligned}
c \theta \int_{\Omega_{1}} h^{2}\left(u_{t}\right) d x & \leqslant c \theta \int_{\Omega_{1}}\left[u_{t} h\left(u_{t}\right)\right]^{\frac{1}{p}} d x \leqslant c \theta\left[\int_{\Omega_{1}} u_{t} h\left(u_{t}\right) d x\right]^{\frac{1}{p}} \\
& \leqslant c \theta^{\frac{p-1}{p}}\left[-E^{\prime}(t)\right]^{\frac{1}{p}} .
\end{aligned}
$$

- Case 1. $p=1$ : Estimate (3.14) yields

$$
\begin{aligned}
F^{\prime}(t) & \leqslant-m \theta(t) E(t)+c \theta(t) \int_{\Gamma_{1}}\left(k_{1}^{\prime \prime} \circ u\right)(t) d \Gamma+c \theta(t) \int_{\Gamma_{1}} k_{2}^{\prime \prime} \circ \frac{\partial u}{\partial v}-c E^{\prime}(t) \\
& \leqslant-m \theta(t) E(t)-c E^{\prime}(t), \quad \forall t \geqslant t_{1},
\end{aligned}
$$

which gives

$$
(F+c E)^{\prime}(t) \leqslant-m \theta(t) E(t), \quad \forall t \geqslant t_{1} .
$$

Hence, using the fact that $F+c E \sim E$, we easily obtain

$$
E(t) \leqslant c^{\prime} e^{-c \int_{0}^{t} \theta(s) d s}=c^{\prime} G^{-1}\left(c \int_{0}^{t} \theta(s) d s\right)
$$

- Case $2.1<p<\frac{3}{2}$ : One can easily show that $\int_{0}^{+\infty}\left[-k_{i}^{\prime}(s)\right]^{1-\delta_{0}} d s<+\infty$ for any $\delta_{0}<2-p$ and $i=1,2$. Using this fact (3.1), and the trace theory and choosing $t_{1}$ even larger if needed, we deduce that, for all $t \geqslant t_{1}$,

$$
\begin{aligned}
\eta(t) & :=\int_{t_{1}}^{t}\left[-k_{1}^{\prime}(s)\right]^{1-\delta_{0}} \int_{\Gamma_{1}}|u(t)-u(t-s)|^{2} d \Gamma d s \\
& \leqslant 2 \int_{t_{1}}^{t}\left[-k_{1}^{\prime}(s)\right]^{1-\delta_{0}} \int_{\Gamma_{1}}\left(|u(t)|^{2}+|u(t-s)|^{2}\right) d \Gamma d s
\end{aligned}
$$




$$
\leqslant c E(0) \int_{t_{1}}^{t}\left[-k_{1}^{\prime}(s)\right]^{1-\delta_{0}} d s<1
$$

and

$$
\begin{aligned}
\gamma(t) & :=\int_{t_{1}}^{t}\left[-k_{2}^{\prime}(s)\right]^{1-\delta_{0}} \int_{\Gamma_{1}}\left|\frac{\partial u(t)}{\partial v}-\frac{\partial u(t-s)}{\partial v}\right|^{2} d \Gamma d s \\
& \leqslant 2 \int_{t_{1}}^{t}\left[-k_{2}^{\prime}(s)\right]^{1-\delta_{0}} \int_{\Gamma_{1}}\left|\frac{\partial u(t)}{\partial v}\right|^{2}+\left|\frac{\partial u(t-s)}{\partial v}\right|^{2} d \Gamma d s \\
& \leqslant c E(0) \int_{t_{1}}^{t}\left[-k_{2}^{\prime}(s)\right]^{1-\delta_{0}} d s<1 .
\end{aligned}
$$

Then, Jensen's inequality (3.1), hypothesis (A2), and (3.15) lead to

$$
\begin{aligned}
& -\int_{t_{1}}^{t} k_{1}^{\prime}(s) \int_{\Gamma_{1}}|u(t)-u(t-s)|^{2} d \Gamma d s \\
& \quad=\int_{t_{1}}^{t}\left[-k_{1}^{\prime}(s)\right]^{\delta_{0}}\left[-k_{1}^{\prime}(s)\right]^{1-\delta_{0}} \int_{\Gamma_{1}}|u(t)-u(t-s)|^{2} d \Gamma d s \\
& \quad=\int_{t_{1}}^{t}\left[-k_{1}^{\prime}(s)\right]^{\left(p-1+\delta_{0}\right)\left(\frac{\delta_{0}}{p-1+\delta_{0}}\right)}\left[-k_{1}^{\prime}(s)\right]^{1-\delta_{0}} \int_{\Gamma_{1}}|u(t)-u(t-s)|^{2} d \Gamma d s \\
& \leqslant \eta(t)\left[\frac{1}{\eta(t)} \int_{t_{1}}^{t}\left[-k_{1}^{\prime}(s)\right]^{\left(p-1+\delta_{0}\right)}\left[-k_{1}^{\prime}(s)\right]^{1-\delta_{0}} \int_{\Gamma_{1}}|u(t)-u(t-s)|^{2} d \Gamma d s\right]^{\frac{\delta_{0}}{p-1+\delta_{0}}} \\
& \quad \leqslant\left[\int_{t_{1}}^{t}\left[-k_{1}^{\prime}(s)\right]^{p} \int_{\Gamma_{1}}|u(t)-u(t-s)|^{2} d \Gamma d s\right]^{\frac{\delta_{0}}{p-1+\delta_{0}}} \\
& \quad \leqslant c\left[\int_{t_{1}}^{t} k_{1}^{\prime \prime}(s) \int_{\Gamma_{1}}|u(t)-u(t-s)|^{2} d \Gamma d s\right]^{\frac{\delta_{0}}{p-1+\delta_{0}}} \leqslant c\left[-E^{\prime}(t)\right]^{\frac{\delta_{0}}{p-1+\delta_{0}}}
\end{aligned}
$$

Similarly

$$
-\int_{t_{1}}^{t} k_{2}^{\prime}(s) \int_{\Gamma_{1}}\left|\frac{\partial u(t)}{\partial v}-\frac{\partial u(t-s)}{\partial v}\right|^{2} d \Gamma d s \leqslant c\left[-E^{\prime}(t)\right]^{\frac{\delta_{0}}{p-1+\delta_{0}}} .
$$

Then, particularly for $\delta_{0}=\frac{1}{2}$, we find that (3.14) becomes

$$
F^{\prime}(t) \leqslant-m \theta E(t)+c \theta\left[-E^{\prime}(t)\right]^{\frac{1}{2 p-1}}+c \theta^{\frac{p-1}{p}}\left[-E^{\prime}(t)\right]^{\frac{1}{p}} .
$$

Now, we multiply by $E^{2 p-2}(t)$ to get, using (3.1),

$$
\left(F E^{2 p-2}\right)^{\prime} \leqslant F^{\prime}(t) E^{2 p-2} \leqslant-m \theta E^{2 p-1}+c \theta E^{2 p-2}\left[-E^{\prime}\right]^{\frac{1}{2 p-1}}+c \theta^{\frac{p-1}{p}} E^{2 p-2}\left[-E^{\prime}\right]^{\frac{1}{p}} .
$$


Then, Young's inequality gives

$$
\left(F E^{2 p-2}\right)^{\prime} \leqslant-m \theta E^{2 p-1}(t)+\varepsilon \theta E^{2 p-1}(t)+C_{\varepsilon} \theta\left(-E^{\prime}(t)\right)+\delta \theta E^{2 p}+C_{\delta}\left(-E^{\prime}(t)\right) .
$$

Consequently, as $E^{2 p}(t) \leqslant E(0) E^{2 p-1}(t)$, picking $\varepsilon+\delta E(0)<m$, we obtain

$$
F_{0}^{\prime}(t) \leqslant-m^{\prime} \theta(t) E^{2 p-1}(t),
$$

where $F_{0}=F E^{2 p-2}+C E \sim E$. Hence we have, for some $a_{0}>0$,

$$
F_{0}^{\prime}(t) \leqslant-a_{0} \theta(t) F_{0}^{2 p-1}(t)
$$

from which we easily deduce that

$$
E(t) \leqslant \frac{a}{\left(a^{\prime} \int_{0}^{t} \theta(s) d s+a^{\prime \prime}\right)^{\frac{1}{2 p-2}}} .
$$

By recalling that $p<3 / 2$ and using (3.17), we find that $\int_{0}^{+\infty} \theta(s) E(s) d s<+\infty$. Hence, by noting that

$$
\begin{aligned}
& \theta(t) \int_{0}^{t} \int_{\Gamma_{1}}|u(t)-u(t-s)|^{2} d \Gamma d s \leqslant c \int_{0}^{t} \theta(s) E(s) d s, \\
& \theta(t) \int_{0}^{t} \int_{\Gamma_{1}}\left|\frac{\partial u(t)}{\partial v}-\frac{\partial u(t-s)}{\partial v}\right|^{2} d \Gamma d s \leqslant c \int_{0}^{t} \theta(s) E(s) d s,
\end{aligned}
$$

estimate (3.14) gives

$$
\begin{aligned}
F^{\prime}(t) \leqslant & -m \theta E(t)+c \theta \int_{\Gamma_{1}}\left(\left[-k_{1}^{\prime}\right]^{p \cdot \frac{1}{p}} \circ u\right)(t) d \Gamma+c \theta \int_{\Gamma_{1}}\left(\left[-k_{2}^{\prime}\right]^{p \cdot \frac{1}{p}} \circ \frac{\partial u}{\partial v}\right)(t) d \Gamma \\
& +c \theta^{\frac{p-1}{p}}\left[-E^{\prime}(t)\right]^{\frac{1}{p}} \\
\leqslant & -m \theta E(t)+c \theta^{\frac{1}{p}}\left[\int_{\Gamma_{1}}\left(\left[-k_{1}^{\prime}\right]^{p} \circ u\right)(t) d \Gamma\right]^{\frac{1}{p}} \\
& +c \theta^{\frac{1}{p}}\left[\int_{\Gamma_{1}}\left(\left[-k_{2}^{\prime}\right]^{p} \circ \frac{\partial u}{\partial v}\right)(t) d \Gamma\right]^{\frac{1}{p}}+c \theta^{\frac{p-1}{p}}\left[-E^{\prime}(t)\right]^{\frac{1}{p}} \\
\leqslant & -m \theta E(t)+c \theta^{\frac{p-1}{p}}\left[\int_{\Gamma_{1}}\left(k_{1}^{\prime \prime} \circ u\right)(t) d \Gamma\right]^{\frac{1}{p}}+c \theta^{\frac{p-1}{p}}\left[\int_{\Gamma_{1}}\left(k_{2}^{\prime \prime} \circ \frac{\partial u}{\partial v}\right)(t) d \Gamma\right]^{\frac{1}{p}} \\
& +c \theta^{\frac{p-1}{p}}\left[-E^{\prime}(t)\right]^{\frac{1}{p}} \\
\leqslant & -m \theta E(t)+c \theta^{\frac{p-1}{p}}\left[-E^{\prime}(t)\right]^{\frac{1}{p}} .
\end{aligned}
$$


Therefore, repeating the above steps, with multiplying by $E^{p-1}(t)$, we arrive at

$$
E(t) \leqslant \frac{a}{\left(a^{\prime} \int_{0}^{t} \theta(s) d s+a^{\prime \prime}\right)^{\frac{1}{p-1}}}=c G^{-1}\left(c^{\prime} \int_{0}^{t} \theta(s) d s+c^{\prime \prime}\right) .
$$

(II) The general case: We define $I(t)$ by

$$
I(t):=\int_{t_{1}}^{t} \frac{-k_{1}^{\prime}(s)}{H_{0}^{-1}\left(k_{1}^{\prime \prime}(s)\right)} \int_{\Gamma_{1}}|u(t)-u(t-s)|^{2} d \Gamma d s,
$$

where $H_{0}$ is such that (2.8) is satisfied. As in (3.15), we find that $I(t)$ satisfies, for all $t \geqslant t_{1}$,

$$
I(t)<1
$$

We also assume, without loss of generality that $I(t)>0$, for all $t>t_{1}$; otherwise (3.14) yields an exponential decay. In addition, we define $\xi(t)$ by

$$
\xi(t):=\int_{t_{1}}^{t} k_{1}^{\prime \prime}(s) \frac{-k_{1}^{\prime}(s)}{H_{0}^{-1}\left(k_{1}^{\prime \prime}(s)\right)} \int_{\Gamma_{1}}|u(t)-u(t-s)|^{2} d \Gamma d s
$$

and infer from (A2) and the properties of $H_{0}$ and $D$ that

$$
\frac{-k_{i}^{\prime}(s)}{H_{0}^{-1}\left(k_{i}^{\prime \prime}(s)\right)} \leqslant \frac{-k_{i}^{\prime}(s)}{H_{0}^{-1}\left(H\left(-k_{i}^{\prime}(s)\right)\right)}=\frac{-k_{i}^{\prime}(s)}{D^{-1}\left(-k_{i}^{\prime}(s)\right)} \leqslant k_{0} \quad \forall i=1,2
$$

for some positive constant $k_{0}$. Then, using (3.1) and choosing $t_{1}$ even larger (if needed), one can easily see that $\xi(t)$ satisfies, for all $t>t_{1}$,

$$
\begin{aligned}
\xi(t) & \leqslant k_{0} \int_{t_{1}}^{t} k_{1}^{\prime \prime}(s) \int_{\Gamma_{1}}|u(t)-u(t-s)|^{2} d \Gamma d s \\
& \leqslant c E(0) \int_{t_{1}}^{t} k_{1}^{\prime \prime}(s) \leqslant-c k_{1}^{\prime}\left(t_{1}\right) E(0) \\
& <\frac{1}{3} \min \left\{r, H(r), H_{0}(r)\right\} .
\end{aligned}
$$

Since $H_{0}$ is strictly convex on $(0, r]$ and $H_{0}(0)=0$, then

$$
H_{0}(\mu x) \leqslant \mu H_{0}(x)
$$

provided $0 \leqslant \mu \leqslant 1$ and $x \in(0, r]$. The use of this fact, hypothesis (A2), (2.10), (3.18), (3.19), and Jensen's inequality leads to

$$
\xi(t)=\frac{1}{I(t)} \int_{t_{1}}^{t} I(t) H_{0}\left[H_{0}^{-1}\left(k_{1}^{\prime \prime}(s)\right)\right] \frac{-k_{1}^{\prime}(s)}{H_{0}^{-1}\left(k_{1}^{\prime \prime}(s)\right)} \int_{\Gamma_{1}}|u(t)-u(t-s)|^{2} d \Gamma d s
$$




$$
\begin{aligned}
& \geqslant \frac{1}{I(t)} \int_{t_{1}}^{t} H_{0}\left[I(t) H_{0}^{-1}\left(k_{1}^{\prime \prime}(s)\right)\right] \frac{-k_{1}^{\prime}(s)}{H_{0}^{-1}\left(k_{1}^{\prime \prime}(s)\right)} \int_{\Gamma_{1}}|u(t)-u(t-s)|^{2} d \Gamma d s \\
& \geqslant H_{0}\left(\frac{1}{I(t)} \int_{t_{1}}^{t} I(t) H_{0}^{-1}\left(k_{1}^{\prime \prime}(s)\right) \frac{-k_{1}^{\prime}(s)}{H_{0}^{-1}\left(k_{1}^{\prime \prime}(s)\right)} \int_{\Gamma_{1}}|u(t)-u(t-s)|^{2} d \Gamma d s\right) \\
& =H_{0}\left(-\int_{t_{1}}^{t} k_{1}^{\prime}(s) \int_{\Gamma_{1}}|u(t)-u(t-s)|^{2} d \Gamma d s\right) .
\end{aligned}
$$

This implies that

$$
-\int_{t_{1}}^{t} k_{1}^{\prime}(s) \int_{\Gamma_{1}}|u(t)-u(t-s)|^{2} d \Gamma d s \leqslant H_{0}^{-1}(\xi(t)) .
$$

We also define

$$
\begin{aligned}
\phi(t) & :=\int_{t_{1}}^{t} \frac{-k_{2}^{\prime}(s)}{H_{0}^{-1}\left(k_{2}^{\prime \prime}(s)\right)} \int_{\Gamma_{1}}\left|\frac{\partial u(t)}{\partial v}-\frac{\partial u(t-s)}{\partial v}\right|^{2} d \Gamma d s \\
\chi(t) & :=\int_{t_{1}}^{t} k_{2}^{\prime \prime}(s) \frac{-k_{2}^{\prime}(s)}{H_{0}^{-1}\left(k_{2}^{\prime \prime}(s)\right)} \int_{\Gamma_{1}}\left|\frac{\partial u(t)}{\partial v}-\frac{\partial u(t-s)}{\partial v}\right|^{2} d \Gamma d s .
\end{aligned}
$$

We similarly deduce, for all $t>t_{1}$, that

$$
\phi(t)<1
$$

and

$$
\chi(t)<\frac{1}{3} \min \left\{r, H(r), H_{0}(r)\right\} .
$$

Repeating the above steps, we arrive at

$$
-\int_{t_{1}}^{t} k_{2}^{\prime}(s) \int_{\Gamma_{1}}\left|\frac{\partial u(t)}{\partial v}-\frac{\partial u(t-s)}{\partial v}\right|^{2} d \Gamma d s \leqslant H_{0}^{-1}(\chi(t)) .
$$

Now we estimate the last integral in (3.14). First, we can assume that $r$ is small enough such that

$$
\operatorname{sh}(s) \leqslant \frac{1}{3} \min \left\{r, H(r), H_{0}(r)\right\} \quad \text { for all }|s| \leqslant r .
$$

Then, with $S(t)$ defined by

$$
S(t):=\frac{1}{\left|\Omega_{1}\right|} \int_{\Omega_{1}} u_{t} h\left(u_{t}\right) d x .
$$


(A3) and Jensen's inequality give

$$
H^{-1}(S(t)) \geqslant c \int_{\Omega_{1}} H^{-1}\left(u_{t} h\left(u_{t}\right)\right) d x \geqslant c \int_{\Omega_{1}} h^{2}\left(u_{t}\right) d x .
$$

Inserting the estimates (3.20), (3.22), and (3.24) into (3.14), we obtain

$$
F^{\prime}(t) \leqslant-m \theta(t) E(t)+c \theta(t)\left[H_{0}^{-1}(\xi(t))+H_{0}^{-1}(\chi(t))+H^{-1}(S(t))\right], \quad \forall t \geqslant t_{1}
$$

One can easily make use of the properties of $H, D, H_{0}$ and the fact that $H_{0}^{-1}(S(t))=$ $D^{-1}\left(H^{-1}(S(t))\right), D^{-1}(0)=0$, and $H^{-1}(S(t)) \leqslant r$ to deduce, for some positive constant $c$, that $H^{-1}(S(t)) \leqslant c H_{0}^{-1}(S(t))$. Therefore

$$
\begin{aligned}
F^{\prime}(t) & \leqslant-m \theta(t) E(t)+c \theta(t)\left[H_{0}^{-1}(\xi(t))+H_{0}^{-1}(\chi(t))+H_{0}^{-1}(S(t))\right] \\
& \leqslant-m \theta(t) E(t)+c \theta(t) H_{0}^{-1}(\xi(t)+\chi(t)+S(t)) .
\end{aligned}
$$

Now, for $\varepsilon_{0}<r$ and $c_{0}>0$, using (3.25), and the fact that $E^{\prime} \leqslant 0, H_{0}^{\prime}>0, H_{0}^{\prime \prime}>0$ on $(0, r]$, we find that the functional $F_{1}$, defined by

$$
F_{1}(t):=H_{0}^{\prime}\left(\varepsilon_{0} \frac{E(t)}{E(0)}\right) F(t)+c_{0} E(t)
$$

satisfies, for some $\alpha_{1}, \alpha_{2}>0$,

$$
\alpha_{1} F_{1}(t) \leqslant E(t) \leqslant \alpha_{2} F_{1}(t)
$$

and, for all $t \geqslant t_{1}$,

$$
\begin{aligned}
F_{1}^{\prime}(t) & =\varepsilon_{0} \frac{E^{\prime}(t)}{E(0)} H_{0}^{\prime \prime}\left(\varepsilon_{0} \frac{E(t)}{E(0)}\right) F(t)+H_{0}^{\prime}\left(\varepsilon_{0} \frac{E(t)}{E(0)}\right) F^{\prime}(t)+c_{0} E^{\prime}(t) \\
& \leqslant-m \theta E(t) H_{0}^{\prime}\left(\varepsilon_{0} \frac{E(t)}{E(0)}\right)+c \theta H_{0}^{\prime}\left(\varepsilon_{0} \frac{E(t)}{E(0)}\right) H_{0}^{-1}(\xi(t)+\chi(t)+S(t))+c_{0} E^{\prime}(t) .
\end{aligned}
$$

Let $H_{0}^{*}$ be the convex conjugate of $H_{0}$ in the sense of Young (see [6] pp. 61-64), then

$$
H_{0}^{*}(s)=s\left(H_{0}^{\prime}\right)^{-1}(s)-H_{0}\left[\left(H_{0}^{\prime}\right)^{-1}(s)\right], \quad \text { if } s \in\left(0, H_{0}^{\prime}(r)\right]
$$

and $H_{0}^{*}$ satisfies the following Young's inequality

$$
A B \leqslant H_{0}^{*}(A)+H_{0}(B), \quad \text { if } A \in\left(0, H_{0}^{\prime}(r)\right], B \in(0, r] .
$$


With $A=H_{0}^{\prime}\left(\varepsilon_{0} \frac{E(t)}{E(0)}\right)$ and $B=H_{0}^{-1}(\xi(t)+\chi(t)+S(t))$, using (3.1), (3.19), (3.21), (3.23), and (3.27)-(3.29), we arrive at

$$
\begin{aligned}
F_{1}^{\prime}(t) \leqslant & -m \theta(t) E(t) H_{0}^{\prime}\left(\varepsilon_{0} \frac{E(t)}{E(0)}\right)+c \theta(t) H_{1}^{*}\left(H_{0}^{\prime}\left(\varepsilon_{0} \frac{E(t)}{E(0)}\right)\right) \\
& +c \theta(t)(\xi(t)+\chi(t)+S(t))+c_{0} E^{\prime}(t) \\
\leqslant & -m \theta(t) E(t) H_{0}^{\prime}\left(\varepsilon_{0} \frac{E(t)}{E(0)}\right)+c \varepsilon_{0} \theta(t) \frac{E(t)}{E(0)} H_{0}^{\prime}\left(\varepsilon_{0} \frac{E(t)}{E(0)}\right)-c E^{\prime}(t)+c_{0} E^{\prime}(t) .
\end{aligned}
$$

Consequently, with a suitable choice of $\varepsilon_{0}$ and $c_{0}$, we obtain, for all $t>t_{1}$,

$$
F_{1}^{\prime}(t) \leqslant-\tau \theta(t)\left(\frac{E(t)}{E(0)}\right) H_{0}^{\prime}\left(\varepsilon_{0} \frac{E(t)}{E(0)}\right)=-\tau \theta(t) H_{2}\left(\frac{E(t)}{E(0)}\right)
$$

where $H_{2}(t)=t H_{0}^{\prime}\left(\varepsilon_{0} t\right)$.

Since $H_{2}^{\prime}(t)=H_{0}^{\prime}\left(\varepsilon_{0} t\right)+\varepsilon_{0} t H_{0}^{\prime \prime}\left(\varepsilon_{0} t\right)$, then, using the strict convexity of $H_{0}$ on $(0, r]$, we find that $H_{2}^{\prime}(t), H_{2}(t)>0$ on $(0,1]$. Thus, with

$$
R(t)=\frac{\alpha_{1} F_{1}(t)}{E(0)}
$$

taking in account (3.26) and (3.30), we have

$$
R(t) \sim E(t)
$$

and, for some $c_{1}>0$,

$$
R^{\prime}(t) \leqslant-c_{1} \theta(t) H_{2}(R(t)), \quad \forall t>t_{1}
$$

Considering $H_{1}(t)=\int_{t}^{1} \frac{1}{H_{2}(s)} d s$, we deduce that $\left(H_{1}(R)\right)^{\prime}(t)>0, \forall t \geqslant t_{1}$, which implies that $H_{1}(R(t)), t \geqslant t_{1}$, is increasing. Thus,

$$
k_{1} \int_{t_{1}}^{t} \theta(s) d s \leqslant \int_{t_{1}}^{t}\left(H_{1}(R)\right)^{\prime}(s) d s \leqslant H_{1}(R(t))-H_{1}\left(R\left(t_{1}\right)\right),
$$

and so, for some $c_{2}>0$,

$$
R(t) \leqslant H_{1}^{-1}\left(c_{1} \int_{t_{1}}^{t} \theta(s) d s+c_{2}\right), \quad \forall t \geqslant t_{1}
$$

Here, we used, based on the properties of $H_{2}$, the fact that $H_{1}$ is strictly decreasing on $(0,1]$. Using (3.31)-(3.32) and by virtue of continuity and boundedness of $E$ and $\theta$, we obtain (2.7). 
Moreover, if $\int_{0}^{1} H_{1}(t) d t<+\infty$, then $\int_{0}^{+\infty} H_{1}^{-1}(t) d t<+\infty$, and so, by $(2.7), \int_{0}^{+\infty} E(t) d t<$ $+\infty$. Then, we have

$$
\begin{aligned}
& \int_{0}^{t} \int_{\Gamma_{1}}|u(t)-u(t-s)|^{2} d \Gamma d s \leqslant c \int_{0}^{t} E(s) d s<+\infty, \\
& \int_{0}^{t} \int_{\Gamma_{1}}\left|\frac{\partial u(t)}{\partial v}-\frac{\partial u(t-s)}{\partial v}\right|^{2} d \Gamma d s \leqslant c \int_{0}^{t} E(s) d s<+\infty .
\end{aligned}
$$

Therefore, we can repeat the same procedures with

$$
\begin{aligned}
I(t) & :=\int_{t_{1}}^{t} \int_{\Gamma_{1}}|u(t)-u(t-s)|^{2} d \Gamma d s, \\
\phi(t) & :=\int_{t_{1}}^{t} \int_{\Gamma_{1}}\left|\frac{\partial u(t)}{\partial v}-\frac{\partial u(t-s)}{\partial v}\right|^{2} d \Gamma d s
\end{aligned}
$$

and

$$
\begin{aligned}
& \xi(t):=\int_{t_{1}}^{t} k_{1}^{\prime \prime}(s) \int_{\Gamma_{1}}|u(t)-u(t-s)|^{2} d \Gamma d s, \\
& \chi(t):=\int_{t_{1}}^{t} k_{2}^{\prime \prime}(s) \int_{\Gamma_{1}}\left|\frac{\partial u(t)}{\partial v}-\frac{\partial u(t-s)}{\partial v}\right|^{2} d \Gamma d s
\end{aligned}
$$

to establish (2.9).

\section{Acknowledgement}

The author thanks University of Sharjah for its continuous support.

\section{References}

[1] F. Alabau-Boussouira, On convexity and weighted integral inequalities for energy decay rates of nonlinear dissipative hyperbolic systems, Appl. Math. Optim. 51 (2005), 61-105. doi:10.1007/s00245.

[2] F. Alabau-Boussouira and P. Cannarsa, A general method for proving sharp energy decay rates for memory-dissipative evolution equations, C. R. Acad. Sci. Paris, Ser. I 347 (2009), 867-872. doi:10.1016/j.crma.2009.05.011.

[3] F. Alabau-Boussouira, P. Cannarsa and D. Sforza, Decay estimates for the second order evolution equation with memory, J. Funct. Anal. 245 (2008), 1342-1372. doi:10.1016/j.jfa.2007.09.012.

[4] K. Ammari and M. Tucsnak, Stabilization of Bernoulli-Euler beam by means of a pointwise feedback force, Siam J. Control Optim. 39(4) (2000), 1160-1181. doi:10.1137/S0363012998349315.

[5] N.E. Amroun and A. Benaissa, Global existence and energy decay of solutions to a Petrovsky equation with general nonlinear dissipation and source term, Georgian Math. J. 13(3) (2006), 397-410.

[6] V.I. Arnold, Mathematical Methods of Classical Mechanics, Springer-Verlag, New York, 1989.

[7] M.M. Cavalcanti, V.N. Domingos Cavalcanti and J.A. Soriano, Global existence and asymptotic stability for the nonlinear and generalized damped extensible plate equation, Commun. Contemp. Math. 6(5) (2004), 705-731. doi:10.1142/ S0219199704001483.

[8] M.M. Cavalcanti and H.P. Oquendo, Frictional versus viscoelastic damping in a semilinear wave equation, SIAM J. Control Optim. 42(4) (2003), 1310-1324. doi:10.1137/S0363012902408010. 
[9] J. Ferreira and S.A. Messaoudic, On the general decay of a nonlinear viscoelastic plate equation with a strong damping and $\vec{p}(x, t)$-Laplacian, Nonlinear Anal. 104 (2014), 40-49. doi:10.1016/j.na.2014.03.010.

[10] A. Guesmia and S.A. Messaoudi, General energy decay estimates of Timoshenko system with frictional versus viscoelastic damping, Math. Methods Appl. Sci. 32(16) (2009), 2102-2122. doi:10.1002/mma.1125.

[11] R.B. Guzman and M. Tucsnak, Energy decay estimates for the damped plate equation with a local degenerated dissipation, Systems \& Control Letters 48 (2003), 191-197. doi:10.1016/S0167-6911(02)00264-5.

[12] X. Han and M. Wang, Energy decay rate for a coupled hyperbolic system with nonlinear damping, Nonlinear Anal. 70 (2009), 3264-3272. doi:10.1016/j.na.2008.04.029.

[13] X. Han and M. Wang, General decay estimate of energy for the second order evolution equation with memory, Acta Appl. Math. 110 (2010), 195-207. doi:10.1007/s10440-008-9397-x.

[14] M.A. Horn, Uniform decay rates for the solution to the Euler Bernoulli plate equation with boundary feedback via bending moments, Differential Integral Equation 6(5) (1992), 1121-1150.

[15] G. Ji and I. Lasiecka, Nonlinear boundary feedback stabilization for a semilinear Kirchhoff plates with dissipation acting only via moments-limiting behavior, J. Math. Anal. Appl. 229 (1999), 452-479. doi:10.1006/jmaa.1998.6170.

[16] J.R. Kang, Energy decay rates for von Karman system with memory and boundary feedback, Appl. Math. Comput. 218 (2012), 9085-9094.

[17] J.R. Kang, General decay for Kirchoff plates with a boundary condition of memory type, Boundary Value Problems 2012 (2012), 129. doi:10.1186/1687-2770-2012-129.

[18] V. Komornik, Decay estimates for a Petrovski system with a nonlinear distributed feedback, IMA Preprints Series (1992), 1083.

[19] V. Komornik, On the nonlinear boundary stabilization of Kirchhoff plates, Nonlinear Differential Equation Appl. 1 (1994), 323-337. doi:10.1007/BF01194984.

[20] J.E. Lagnese, Asymptotic Energy Estimates for Kirchoff Plates Subject to Weak Viscoelastic Damping, International Series of Numerical Mathematics, Vol. 91, Birkhauser-Verlag, Bassel, 1989.

[21] J.E. Lagnese, Boundary stabilization of thin plates, SIAM, Philadelphia, PA, 1989.

[22] I. Lasiecka, Exponential decay rates for the solutions of Euler-Bernoulli moments only, J. Differential Equations 95 (1992), 169-182. doi:10.1016/0022-0396(92)90048-R.

[23] I. Lasiecka and D. Tataru, Uniform boundary stabilization of semilinear wave equation with nonlinear boundary damping, Differential Integral Equations 8 (1993), 507-533.

[24] W.-J. Liu and E. Zuazua, Decay rates for dissipative wave equations, Ricerche Mat. 48 (1999), 61-75.

[25] W.J. Liu, General decay rate estimate for a viscoelastic equation with weakly nonlinear time-dependent dissipation and source terms, J. Math. Phys. 50(11) (2009), 113506. doi:10.1063/1.3254323.

[26] P. Martinez, A new method to obtain decay rate estimates for dissipative systems, ESAIM Control Optim. Calc. Var. 4 (1999), 419-444. doi:10.1051/cocv:1999116.

[27] P. Martinez, A new method to obtain decay rate estimates for dissipative systems with localized damping, Rev. Mat. Complut. 12(1) (1999), 251-283.

[28] S.A. Messaoudi, On the control of solutions of a viscoelastic equations, J. Franklin Inst. 334 (2007), $765-776$. doi:10.1016/j.jfranklin.2006.02.029.

[29] S.A. Messaoudi, General decay of solutions of a viscoelastic equation, J. Math. Anal. Appl. 341 (2008), 1457-1467. doi:10.1016/j.jmaa.2007.11.048.

[30] S.A. Messaoudi and M.I. Mustafa, A stability result in a memory-type Timoshenko system, Dynam. Systems Appl. 18 (2009), 457-468.

[31] S.A. Messaoudi and M.I. Mustafa, On convexity for energy decay rates of a viscoelastic equation with boundary feedback, Nonlinear Anal. TMA 72 (2010), 3602-3611. doi:10.1016/j.na.2009.12.040.

[32] J.E. Munoz Rivera, E.C. Lapa and R. Barreto, Decay rates for viscoelastic plates with memory, J. Elasticity 44 (1996), 61-87. doi:10.1007/BF00042192.

[33] J.E. Munoz Rivera and M.G. Naso, On the decay of the energy for systems with memory and indefinite dissipation, Asympt. Anal. 49(3-4) (2006), 189-204.

[34] J.E. Munoz Rivera, M.G. Naso and F.M. Vegni, Asymptotic behavior of the energy for a class of weakly dissipative second-order systems with memory, J. Math. Anal. Appl. 286(2) (2003), 692-704. doi:10.1016/S0022-247X(03)00511-0.

[35] M.I. Mustafa, Uniform decay for wave equations with weakly dissipative boundary feedback, Dynamical Systems (2015). doi:10.1080/14689367.2014.1002455.

[36] M.I. Mustafa and S.A. Messaoudi, General energy decay rates for a weakly damped wave equation, Commun. Math. Anal. 9(2) (2010), 67-76.

[37] M.I. Mustafa and S.A. Messaoudi, Energy decay rates for a Timoshenko system with vescolastic boundary conditions, Appl. Math. Comput. 218 (2012), 9125-9131.

[38] M.I. Mustafa and S.A. Messaoudi, General stability result for viscoelastic wave equations, J. Math. Phys. 53 (2012), 053702. doi:10.1063/1.4711830. 
[39] A.F. Pazoto, L. Colho and R.C. Charao, Uniform stabilization of a plate equation with nonlinear localized dissipation, Proyecciones (Universidad Catolica del Norte) 23(3) (2004), 205-234.

[40] M.L. Santos and F. Junior, A boundary condition with memory for Kirchoff plates equations, Appl. Math. Comput. 148 (2004), 475-496.

[41] C.F. Vasconcellos and L.M. Teixeira, Existence, uniqueness and stabilization for a nonlinear plate system with nonlinear damping, Annales de Toulouse 8(1) (1999), 173-193. doi:10.5802/afst.928. 